\title{
Asymptotic Approximations in Quantum Calculus
}

\author{
Ahmed FITOUHI ${ }^{a}$, Kamel BRAHIM $^{b}$ and Néji BETTAIBI ${ }^{c}$ \\ ${ }^{a}$ Faculté des Sciences de Tunis, 1060 Tunis, Tunisia. \\ E-mail: Ahmed.Fitouhi@fst.rnu.tn \\ ${ }^{b}$ Institut Préparatoire aux Études d'Ingénieur de Tunis, Tunisia. \\ E-mail: Kamel.Brahim@ipeit.rnu.tn \\ ${ }^{c}$ Institut Préparatoire aux Études d'Ingénieur de Nabeul, 8000 Nabeul, Tunisia. \\ E-mail: Neji.Bettaibi@ipein.rnu.tn
}

Received April 18, 2005; Accepted in Revised Form June 7, 2005

\begin{abstract}
This paper aims to study the asymptotic approximation of some functions defined by the $q$-Jackson integrals, for a fix $q \in] 0,1[$. For this purpose, we shall attempt to extend the classical methods by giving their $q$-analogues. In particular, a $q$-analogue of the Watson's lemma is discussed and new asymptotic expansions of the $q-j_{\alpha}$ Bessel function and of the $q$-complementary error function are established.
\end{abstract}

\section{Introduction}

The notion of asymptotic expansion was introduced by Poincaré in the 19th century and developed by both mathematicians and physicists, such as Riemann, Laplace, Stokes and Kelven, etc. So, different definitions and different notations were introduced. In this paper we will use the Poincaré's definition and the Landau's notations. This concept enables one to obtain numerical as well as qualitative results for many problems. When modeling physical phenomena, it is often useful to know the asymptotic behavior of functions defined by integrals. For example, many special functions have integral representations, as they are solutions of various kinds of differential equations. Also, if we use Laplace, Fourier or Hankel transformations to solve differential equations, we are often left with an integral representation of the solution. In classical analysis, many techniques and methods were introduced to derive asymptotic expansions of such functions. In this paper, we shall attempt to extend the classical theory and we shall try to derive asymptotic expansions of functions defined with the help of the $q$-Jackson integral. We are not in a situation to claim that all our results are new, but the methods used are direct and constructive, and have a good resemblance with the classical ones.

This paper is organized as follows: in Sec. 2, we present some preliminaries results and notations that will be useful in the sequel. In Sec. 3, we discuss the method of $q$ integration by parts, through three examples. In Sec. 4, we state the asymptotic behavior 
of the Laplace $q$-integral and we give the $q$-analogue of the Watson's lemma. In Sec. 5, we discuss the asymptotic behavior of the Fourier $q$-integral. Finally, in Sec. 6, we discuss the asymptotic expansion of the third $q$-Bessel function and considering the asymptotic behavior at 0 of a function $f$, we give an asymptotic expansion of its $q$-Hankel transform and $q$-Cosine Fourier transform.

\section{Notation and preliminaries}

Throughout this paper, we will fix $q \in] 0,1[$. We recall some usual notions and notations used in the $q$-theory (see [8] and [11]).

Let $a \in \mathbb{C}$, the $q$-shifted factorial are defined by

$$
\begin{aligned}
& (a ; q)_{0}=1, \quad(a ; q)_{n}=\prod_{k=0}^{n-1}\left(1-a q^{k}\right), \quad n=1,2, \ldots \\
& (a ; q)_{\infty}=\lim _{n \rightarrow+\infty}(a ; q)_{n}=\prod_{k=0}^{\infty}\left(1-a q^{k}\right) .
\end{aligned}
$$

We also denote

$$
\begin{aligned}
& \left(a_{1}, a_{2}, \ldots, a_{p} ; q\right)_{n}=\left(a_{1} ; q\right)_{n}\left(a_{2} ; q\right)_{n} \ldots\left(a_{p} ; q\right)_{n}, \quad n=0,1,2,3, \ldots \infty \\
& {[x]_{q}=\frac{1-q^{x}}{1-q}, \quad x \in \mathbb{C}}
\end{aligned}
$$

and

$$
[n]_{q} !=\frac{(q ; q)_{n}}{(1-q)^{n}}, \quad n \in \mathbb{N} .
$$

The $q$-derivative $D_{q} f$ of a function $f$ is given by

$$
\left(D_{q} f\right)(x)=\frac{f(x)-f(q x)}{(1-q) x}, \quad \text { if } \quad x \neq 0,
$$

$\left(D_{q} f\right)(0)=f^{\prime}(0)$ provided $f^{\prime}(0)$ exists. If $f$ is differentiable then $\left(D_{q} f\right)(x)$ tends to $f^{\prime}(x)$ as $q$ tends to 1 .

For $n \in \mathbb{N}$, we note

$$
D_{q}^{1}=D_{q} \quad \text { and } \quad D_{q}^{n}=D_{q}\left(D_{q}^{n-1}\right) .
$$

We remark that

$$
D_{q}(f . g)(x)=g(x) D_{q} f(x)+f(q x) D_{q} g(x)
$$

and if $f$ is $n$ times continuously differentiable near 0 , then (see also [3]),

$$
D_{q}^{n} f(0)=\frac{[n]_{q} !}{n !} f^{(n)}(0)
$$


The $q$-Jackson integrals from 0 to $a$ and from 0 to $\infty$ are defined by (see [10])

$$
\begin{aligned}
& \int_{0}^{a} f(x) d_{q} x=(1-q) a \sum_{n=0}^{\infty} f\left(a q^{n}\right) q^{n}, \\
& \int_{0}^{\infty} f(x) d_{q} x=(1-q) \sum_{n=-\infty}^{\infty} f\left(q^{n}\right) q^{n},
\end{aligned}
$$

provided the sums converge absolutely.

The $q$-Jackson integral in a generic interval $[a, b]$ is given by (see [10])

$$
\int_{a}^{b} f(x) d_{q} x=\int_{0}^{b} f(x) d_{q} x-\int_{0}^{a} f(x) d_{q} x .
$$

The improper integral is defined in the following way (see [13])

$$
\int_{0}^{\frac{\infty}{A}} f(x) d_{q} x=(1-q) \sum_{n=-\infty}^{\infty} f\left(\frac{q^{n}}{A}\right) \frac{q^{n}}{A} .
$$

We remark that for $n \in \mathbb{Z}$, we have

$$
\int_{0}^{\frac{\infty}{q^{n}}} f(x) d_{q} x=\int_{0}^{\infty} f(x) d_{q} x .
$$

The $q$-integration by parts is given for suitable functions $f$ and $g$ by (see [1], [11])

$$
\int_{a}^{b} g(x) D_{q} f(x) d_{q} x=f(b) g(b)-f(a) g(a)-\int_{a}^{b} f(q x) D_{q} g(x) d_{q} x .
$$

Definition 1. A function $f$ is $q$-integrable on $\left[0, \infty\left[\right.\right.$ if the series $\sum_{n \in \mathbb{Z}} q^{n} f\left(q^{n}\right)$ converges absolutely.

We write $L^{1}\left(\mathbb{R}_{q,+}\right)$ the set of all functions that are $q$-integrable on $\left[0, \infty\left[\right.\right.$, where $\mathbb{R}_{q,+}$ is the set:

$$
\mathbb{R}_{q,+}=\left\{q^{n}: n \in \mathbb{Z}\right\}
$$

Proposition 1. (see [1], [3] or [11]) The q-analogue of the integration theorem by change of variable is given when $u(x)=\alpha x^{\beta}, \quad \alpha \in \mathbb{C}$ and $\beta>0$ as follows

$$
\int_{u(a)}^{u(b)} f(u) d_{q} u=\int_{a}^{b} f(u(x)) D_{q^{\frac{1}{\beta}}} u(x) d_{q^{\frac{1}{\beta}}} x .
$$

The $q$-analogues of the exponential function (see [8], [11]) are given by

$$
E_{q}^{z}={ }_{0} \varphi_{0}(-;-; q,-(1-q) z)=\sum_{n=0}^{\infty} q^{\frac{n(n-1)}{2}} \frac{z^{n}}{[n]_{q} !}=(-(1-q) z ; q)_{\infty}
$$




$$
e_{q}^{z}={ }_{1} \varphi_{0}(0 ;-; q,(1-q) z)=\sum_{n=0}^{\infty} \frac{z^{n}}{[n]_{q} !}=\frac{1}{((1-q) z ; q)_{\infty}} .
$$

For the convergence of the second series, we need $|z|<(1-q)^{-1}$; however, because of its product representation, $e_{q}$ is continuable to a meromorphic function on $\mathbb{C}$ and has simple poles at $z=q^{-n}(1-q)^{-1}, \quad n \in \mathbb{N}$. They satisfy the relations (see [11])

$$
D_{q} e_{q}^{z}=e_{q}^{z}, \quad D_{q} E_{q}^{z}=E_{q}^{q z}
$$

and

$$
E_{q}^{-z} e_{q}^{z}=e_{q}^{z} E_{q}^{-z}=1 .
$$

Jackson [10] defined the $q$-analogue of the Gamma function by

$$
\Gamma_{q}(x)=\frac{(q ; q)_{\infty}}{\left(q^{x} ; q\right)_{\infty}}(1-q)^{1-x}, \quad x \neq 0,-1,-2, \ldots
$$

It is well-known that it satisfies for $\Re(x)>0$,

$$
\Gamma_{q}(x+1)=[x]_{q} \Gamma_{q}(x), \quad \Gamma_{q}(1)=1 \quad \text { and } \quad \lim _{q \rightarrow 1^{-}} \Gamma_{q}(x)=\Gamma(x) .
$$

Moreover, it has the $q$-integral representations (see [1])

$$
\Gamma_{q}(s)=\int_{0}^{\frac{1}{1-q}} t^{s-1} E_{q}^{-q t} d_{q} t=\int_{0}^{\frac{\infty}{1-q}} t^{s-1} E_{q}^{-q t} d_{q} t .
$$

Now, we give a new proof of the following result due to De Sole and Kac (see [1]).

Proposition 2. We have

$$
\Gamma_{q}(s)=K_{q}(s) \int_{0}^{\frac{\infty}{1-q}} x^{s-1} e_{q}^{-x} d_{q} x, \quad \Re(s)>0,
$$

where

$$
K_{q}(t)=\frac{(-q,-1 ; q)_{\infty}}{\left(-q^{t},-q^{1-t} ; q\right)_{\infty}}, \quad \Re(s)>0 .
$$

Proof. Using the Ramanujan's summation formula for ${ }_{1} \psi_{1}(a, b ; q, z)$ (see [8] and [11]) and simple calculus, we obtain for $s \in \mathbb{C}$, with $\Re(s)>0$,

$$
\begin{aligned}
\int_{0}^{\frac{\infty}{1-q}} t^{s-1} e_{q}^{-t} d_{q} t & =(1-q) \sum_{n=-\infty}^{\infty} e_{q}^{-\frac{q^{n}}{1-q}}\left(\frac{q^{n}}{1-q}\right)^{s}=(1-q)^{1-s} \sum_{n=-\infty}^{\infty} \frac{q^{n s}}{\left(-q^{n} ; q\right)_{\infty}} \\
& =\frac{(1-q)^{1-s}}{(-1 ; q)_{\infty}} \sum_{n=-\infty}^{\infty}(-1 ; q)_{n} q^{n s}=\frac{(1-q)^{1-s}}{(-1 ; q)_{\infty}}{ }_{1} \psi_{1}\left(-1 ; 0 ; q, q^{s}\right) \\
& =\frac{(1-q)^{1-s}}{(-1 ; q)_{\infty}} \frac{\left(q,-q^{s},-q^{1-s} ; q\right)_{\infty}}{\left(-q, q^{s} ; q\right)_{\infty}}=\frac{\Gamma_{q}(s)}{K_{q}(s)},
\end{aligned}
$$

which achieves the proof. 
Remark. Additionally, if $\log (1-q)(\log (q))^{-1} \in \mathbb{Z}$, we obtain

$$
\Gamma_{q}(s)=K_{q}(s) \int_{0}^{\infty} x^{s-1} e_{q}^{-x} d_{q} x=\int_{0}^{\infty} t^{s-1} E_{q}^{-q t} d_{q} t, \quad \Re(s)>0,
$$

where $\log (x)$ means $\log _{e}(x)$.

We recall that the $q$-hypergeometric function ${ }_{1} \varphi_{1}$ satisfies the following properties (see [6] and [14])

1. For all $w, z \in \mathbb{C}$, we have

$$
(w, q)_{\infty 1} \varphi_{1}(0 ; w ; q ; z)=(z, q)_{\infty} 1 \varphi_{1}(0 ; z ; q ; w) .
$$

2. For $n \in \mathbb{N}$ and $z \in \mathbb{C}$, we have

$$
\left(q^{1-n} ; q\right)_{\infty 1} \varphi_{1}\left(0 ; q^{1-n} ; q ; z\right)=(-1)^{n} q^{\frac{n(n-1)}{2}} z^{n}\left(q^{n+1} ; q\right)_{\infty 1} \varphi_{1}\left(0 ; q^{n+1} ; q ; q^{n} z\right)
$$

3. Both sides of the above equation are majorized by

$$
q^{\frac{n(n-1)}{2}}|z|^{n}(-|z| ; q)_{\infty}(-q ; q)_{\infty} .
$$

We recall the definition of the $q$-trigonometric functions $q$-cosine and $q$-sine (see [6])

$$
\cos \left(x ; q^{2}\right)={ }_{1} \varphi_{1}\left(0, q ; q^{2},(1-q)^{2} x^{2}\right)=\sum_{n=0}^{\infty}(-1)^{n} q^{n(n-1)} \frac{x^{2 n}}{[2 n]_{q} !}
$$

and

$$
\sin \left(x ; q^{2}\right)=x_{1} \varphi_{1}\left(0, q^{3} ; q^{2},(1-q)^{2} x^{2}\right)=\sum_{n=0}^{\infty}(-1)^{n} q^{n(n-1)} \frac{x^{2 n+1}}{[2 n+1]_{q} !} .
$$

Using the properties of ${ }_{1} \varphi_{1}$ mentioned above, we can easily prove (see [6]) that $q$-cosine and $q$-sine are majorized by $\left((q, q)_{\infty}^{2}\right)^{-1}$ and tend to 0 as $x$ tends to $\infty$. Now, we can recall the $q$-analogue of the Riemann-Lebesgue lemma using the fact that for $x \in \mathbb{R}$,

$$
\left|e_{q}^{i x}\right|^{2} \leq \frac{1}{1+(1-q)^{2} x^{2}}
$$

Lemma 1. If $f$ is $q$-integrable on $[0,+\infty[$, then

$$
\begin{aligned}
\lim _{x \rightarrow+\infty} \int_{0}^{\infty} f(t) e_{q}^{i x t} d_{q} t & =\lim _{x \rightarrow+\infty} \int_{0}^{\infty} f(t) \cos \left(x t, q^{2}\right) d_{q} t \\
& =\lim _{x \rightarrow+\infty} \int_{0}^{\infty} f(t) \sin \left(x t, q^{2}\right) d_{q} t=0 .
\end{aligned}
$$

The second and the third equalities are proved in [6], but the first equality seems new and can be proved in the same way, using (2.31). 


\section{3 q-Integration by parts' method}

The method of $q$-integration by parts is a simple technique for deriving asymptotic expansions of definite $q$-integral. Each $q$-integration produces a term expansion, and the error term is given explicitly as $q$-integral. The following examples can clarify this idea.

\section{Example 1. $q$-Complementary error function}

We define the $q$-Complementary error function as

$$
\operatorname{Err}(x ; q)=\int_{x}^{\infty} e_{q^{2}}^{-t^{2}} d_{q} t
$$

and we state the following proposition:

Proposition 3. For all $n \in \mathbb{N}$, we have

$$
\begin{aligned}
& \operatorname{Err}(x ; q)=\frac{e_{q^{2}}^{-x^{2}}}{[2]_{q} x}+\sum_{k=1}^{n}(-1)^{k} q^{-k(2 k+1)} \frac{[3]_{q}[5]_{q} \ldots[2 k-1]_{q}}{[2]_{q}^{k+1}} \frac{e_{q^{2}}^{-q^{2 k} x^{2}}}{x^{2 k+1}}+o\left(x^{-(2 n+1)}\right), \\
& \text { as } x \rightarrow+\infty .
\end{aligned}
$$

Proof. Using the $q$-integration by parts rule, we obtain

$$
\int_{x}^{\infty} \frac{e_{q^{2}}^{-q^{2 n} t^{2}}}{t^{2 n}} d_{q} t=\frac{1}{q^{2 n}[2]_{q}} \frac{e_{q^{2}}^{-q^{2 n} x^{2}}}{x^{2 n+1}}+\frac{[-(2 n+1)]_{q}}{q^{2 n}[2]_{q}} \int_{x}^{\infty} \frac{e_{q^{2}}^{-q^{2(n+1)} t^{2}}}{t^{2(n+1)}} d_{q} t .
$$

Then, for all $n \in \mathbb{N}$, we obtain

$$
\begin{aligned}
\int_{x}^{\infty} e_{q^{2}}^{-t^{2}} d_{q} t & =\frac{e_{q^{2}}^{-x^{2}}}{[2]_{q} x}+\frac{1}{[2]_{q} x} \sum_{k=1}^{n}(-1)^{k} q^{-k(2 k+1)} \frac{[3]_{q}[5]_{q} \ldots[2 k-1]_{q}}{[2]_{q}^{k}} \frac{e_{q^{2}}^{-q^{2 k} x^{2}}}{x^{2 k}}+ \\
& +(-1)^{n+1} q^{-(n+1)(2 n+1)} \frac{[3]_{q} \ldots[2 n+1]_{q}}{[2]_{q}^{n+1}} \int_{x}^{\infty} \frac{e_{q^{2}}^{-q^{2(n+1)}} t^{2}}{t^{2(n+1)}} d_{q} t \\
& =\frac{e_{q^{2}}^{-x^{2}}}{[2]_{q} x}+\frac{1}{[2]_{q} x} \sum_{k=1}^{n}(-1)^{k} q^{-k(2 k+1)} \frac{[3]_{q}[5]_{q} \ldots[2 k-1]_{q}}{[2]_{q}^{k}} \frac{e_{q^{2}}^{-q^{2 k} x^{2}}}{x^{2 k}}+ \\
& +o\left(\frac{1}{x^{2 n+1}}\right) .
\end{aligned}
$$

(3) is then proved.

\section{Example 2. The $q-j_{\alpha}$ Bessel function}

For $\alpha>-\frac{1}{2}$, the $q-j_{\alpha}$ Bessel function has the following $q$-integral representation of Mehler type (see [7])

$$
j_{\alpha}\left(x ; q^{2}\right)=C\left(\alpha ; q^{2}\right) \int_{0}^{1} f_{0}(t) \cos \left(x t ; q^{2}\right) d_{q} t,
$$

where $f_{0}(t)=\frac{\left(t^{2} q^{2} ; q^{2}\right)_{\infty}}{\left(t^{2} q^{2 \alpha+1} ; q^{2}\right)_{\infty}}$ and $C\left(\alpha ; q^{2}\right)=\frac{(1+q) \Gamma_{q^{2}}(\alpha+1)}{\Gamma_{q^{2}}\left(\frac{1}{2}\right) \Gamma_{q^{2}}\left(\alpha+\frac{1}{2}\right)}$.

We prove the following result. 
Proposition 4. For $n \in \mathbb{N}$, we put $f_{n}=D_{q}^{n} f_{0}$. Then for all $n \in \mathbb{N}^{*}$ and $x \in \mathbb{R}_{q,+}$, we have

$j_{\alpha}\left(x ; q^{2}\right)=C\left(\alpha ; q^{2}\right) \sum_{k=0}^{n-1} \frac{(-1)^{k}}{q^{k^{2}-2 k} x^{2 k}}\left[f_{2 k}(1) \frac{\sin \left(x q^{k} ; q^{2}\right)}{x q^{k}}+q f_{2 k+1}(1) \frac{\cos \left(x q^{k} ; q^{2}\right)}{\left(x q^{k}\right)^{2}}\right]+o\left(x^{-2 n}\right)$.

Proof. It is easy to see that $f_{0}$ is an even function and infinitely differentiable on a neighborhood of 0 . Then $f_{2 k+1}(0)=\frac{[2 k+1]_{q} !}{(2 k+1) !} f_{0}^{(2 k+1)}(0)=0, k=0,1,2, \ldots$.

Two $q$-integrations by parts show for $k=0,1,2, \ldots$,

$$
\begin{aligned}
\int_{0}^{1} f_{2 k}(t) \cos \left(x q^{k} t ; q^{2}\right) d_{q} t & =f_{2 k}(1) \frac{\sin \left(x q^{k} ; q^{2}\right)}{x q^{k}}+q f_{2 k+1}(1) \frac{\cos \left(x q^{k} ; q^{2}\right)}{\left(x q^{k}\right)^{2}}- \\
& -\frac{1}{x^{2} q^{2 k-1}} \int_{0}^{1} f_{2(k+1)}(t) \cos \left(x q^{k+1} t ; q^{2}\right) d_{q} t .
\end{aligned}
$$

Thus by induction, we obtain for $n=0,1,2, \ldots$,

$$
\begin{aligned}
\int_{0}^{1} f_{0}(t) \cos \left(x t ; q^{2}\right) d_{q} t & =\sum_{k=0}^{n-1} \frac{(-1)^{k}}{q^{k^{2}-2 k} x^{2 k}}\left[f_{2 k}(1) \frac{\sin \left(x q^{k} ; q^{2}\right)}{x q^{k}}+q f_{2 k+1}(1) \frac{\cos \left(x q^{k} ; q^{2}\right)}{\left(x q^{k}\right)^{2}}\right] \\
& +\frac{(-1)^{n}}{q^{n^{2}-2 n} x^{2 n}} \int_{0}^{1} f_{2 n}(t) \cos \left(x q^{n} t ; q^{2}\right) d_{q} t .
\end{aligned}
$$

As mentioned above, $\int_{0}^{1} f_{2 n}(t) \cos \left(x q^{n} t ; q^{2}\right) d_{q} t$ tends to 0 as $x$ tends to $+\infty$. This completes the proof.

Example 3. Another type of $q$-integral to which the method of $q$-integration by parts can be applied is the $q$-integral

$$
F(x)=\int_{a}^{b} f(t) e_{q}^{i x t} d_{q} t
$$

where $a, b$ are reals and $f$ is an $n$-times continuously $q$-differentiable function in $[a, b]$. By successive $q$-integration by parts, we have

$$
\begin{aligned}
F(x) & =\sum_{k=0}^{n-1} q^{-\frac{k(k-1)}{2}}\left(\frac{i}{x}\right)^{k+1}\left[D_{q}^{k} f(a) e_{q}^{i x q^{k} a}-D_{q}^{k} f(b) e_{q}^{i x q^{k} b}\right]+ \\
& +q^{-\frac{n(n-1)}{2}}\left(\frac{i}{x}\right)^{n} \int_{a}^{b} D_{q}^{n} f(t) e_{q}^{i x q^{n} t} d_{q} t .
\end{aligned}
$$

On the other hand, the $q$-Riemann-lebesgue lemma gives

$$
\left(\frac{i}{x}\right)^{n} \int_{a}^{b} D_{q}^{n} f(t) e_{q}^{i x q^{n} t} d_{q} t=o\left(x^{-n}\right) \quad \text { as } \quad x \rightarrow+\infty
$$

so

$$
F(x)=\sum_{k=0}^{n-1} q^{-\frac{k(k-1)}{2}}\left(\frac{i}{x}\right)^{k+1}\left[D_{q}^{k} f(a) e_{q}^{i x q^{k} a}-D_{q}^{k} f(b) e_{q}^{i x q^{k} b}\right]+o\left(x^{-n}\right)
$$




\section{Laplace $q$-integrals}

In this section, we define the $q$-integral of Laplace type by

$$
F(x)=\int_{0}^{\infty} f(t) e_{q}^{x \varphi(t)} d_{q} t
$$

where $\varphi$ is a real function and we assume $x>0$. Typically $x$ is a large parameter and we are interested in the asymptotic behavior of $F(x)$ as $x \rightarrow+\infty$.

Proposition 5. Let $f$ be a function defined on $[0, \infty[$, continuous at 0 and verifies for some $\alpha>0$ and $n$ integer,

$$
\int_{0}^{\infty} t^{n}|f(t)| e_{q}^{-\alpha t} d_{q} t<\infty
$$

then

$$
\lim _{x \rightarrow+\infty} x^{n+1} \int_{0}^{\infty} t^{n} f(t) e_{q}^{-x t} d_{q} t=\frac{[n]_{q} !}{q^{\frac{n(n+1)}{2}}} f(0) .
$$

Proof. First, by $q$-integration by parts, we have for all $n \in \mathbb{N}$ and $x>0$,

$$
\int_{0}^{\infty} t^{n} e_{q}^{-x t} d_{q} t=\frac{[n]_{q} !}{q^{\frac{n(n+1)}{2}}} \frac{1}{x^{n+1}} .
$$

Then (4.2) is true for $f$ constant, so it is sufficient to prove the result when $f(0)=0$. For $k \in \mathbb{N}$, we put $x_{k}=q^{-k}(1-q)^{-1}$ and we fix $r \in \mathbb{N}$ such that $x_{r} \geq \alpha$. Let $\varepsilon>0$, since $f$ is continuous at 0 , there exists $p_{0} \in \mathbb{N}$ such that $p_{0} \geq r$ and

$$
\left|f\left(q^{p}\right)\right| \leq \varepsilon, \quad p \geq p_{0} .
$$

For $k \geq p_{0}$, we have

$$
\begin{aligned}
\left|\int_{0}^{\infty} t^{n} f(t) e_{q}^{-x_{k} t} d_{q} t\right| \leq & (1-q) \sum_{p=-\infty}^{p_{0}-1} q^{(n+1) p} e_{q}^{-x_{k} q^{p}}\left|f\left(q^{p}\right)\right|+ \\
& +(1-q) \sum_{p=p_{0}}^{+\infty} q^{(n+1) p} e_{q}^{-x_{k} q^{p}}\left|f\left(q^{p}\right)\right|
\end{aligned}
$$

The second sum of the right hand side of the above inequality is majorized by

$$
\varepsilon \int_{0}^{\infty} t^{n} e_{q}^{-x_{k} t} d_{q} t=\frac{[n]_{q} !}{q^{\frac{n(n+1)}{2}}} \frac{\varepsilon}{x_{k}^{n+1}}
$$

The definition of $x_{r}$ leads to

$$
\int_{0}^{\infty} t^{n}|f(t)| e_{q}^{-x_{r} t} d_{q} t=(1-q) \sum_{p=-\infty}^{\infty} \frac{q^{(n+1) p}\left|f\left(q^{p}\right)\right|}{\left(-q^{p-r} ; q\right)_{\infty}}<\infty .
$$


And the properties of the $q$-shifted factorial give for all $p \leq p_{0}$,

$$
\begin{aligned}
\left(-x_{k}(1-q) q^{p} ; q\right)_{\infty} & =\left(-q^{p-r} ; q\right)_{\infty}\left(-q^{p-k} ; q\right)_{k-r} \\
& \geq\left(-q^{p-r} ; q\right)_{\infty}\left(-q^{p_{0}-k} ; q\right)_{k-r} \\
& \geq\left(-q^{p-r} ; q\right)_{\infty} q^{-\frac{\left(k-p_{0}\right)\left(k-p_{0}+1\right)}{2}} .
\end{aligned}
$$

Together (4.4) and (4.7) yield

$$
\begin{aligned}
(1-q) \sum_{p=-\infty}^{p_{0}-1} q^{(n+1) p} e_{q}^{-x_{k} q^{p}}\left|f\left(q^{p}\right)\right| & =(1-q) \sum_{p=-\infty}^{p_{0}-1} \frac{q^{(n+1) p}\left|f\left(q^{p}\right)\right|}{\left(-(1-q) x_{k} q^{p} ; q\right)_{\infty}} \\
& \leq q^{\frac{\left(k-p_{0}\right)\left(k-p_{0}+1\right)}{2}} \int_{0}^{\infty} t^{n}|f(t)| e_{q}^{-x_{r} t} d_{q} t .
\end{aligned}
$$

Then

$$
x_{k}^{n+1}(1-q) \sum_{p=-\infty}^{p_{0}-1} q^{(n+1) p} e_{q}^{-x_{k} q^{p}}\left|f\left(q^{p}\right)\right| \rightarrow 0 \quad \text { as } \quad k \rightarrow+\infty .
$$

Again, (4.3) and (4.8) yield: $\exists k_{0} \in \mathbb{N}$ such that $\forall k \geq k_{0}$, we have

$$
\left|x_{k}^{n+1} \int_{0}^{\infty} t^{n} f(t) e_{q}^{-x_{k} t} d_{q} t\right| \leq\left(1+\frac{[n]_{q} !}{q^{\frac{n(n+1)}{2}}}\right) \varepsilon .
$$

Thus,

$$
\lim _{k \rightarrow+\infty} x_{k}^{n+1} \int_{0}^{\infty} t^{n} f(t) e_{q}^{-x_{k} t} d_{q} t=0 .
$$

Finally, since for all $x \geq \frac{1}{1-q}$, there exists $k \in \mathbb{N}$ such that

$$
x_{k}=\frac{q^{-k}}{1-q} \leq x \leq x_{k+1}=q^{-1} x_{k},
$$

then,

$$
\lim _{x \rightarrow+\infty} x^{n+1} \int_{0}^{\infty} t^{n} f(t) e_{q}^{-x t} d_{q} t=0 .
$$

The formula (4.2) is then proved.

Using the $q$-Taylor formula (see [2], [3] and [11]) and the above result, we obtain the following

Corollary 1. If $f$ is a n-times continuously q-differentiable function on $[0,+\infty[$, satisfying for some $\alpha>0$, the following condition

$$
\int_{0}^{\infty} e_{q}^{-\alpha t}|f(t)| d_{q} t<\infty
$$

then

$$
\int_{0}^{\infty} e_{q}^{-x t} f(t) d_{q} t=\sum_{k=0}^{n} \frac{D_{q}^{k} f(0)}{q^{\frac{k(k+1)}{2}}} \frac{1}{x^{k+1}}+o\left(\frac{1}{x^{n+1}}\right) .
$$


Proof. According to the $q$-Taylor formula, we have

$$
f(t)=\sum_{k=0}^{n} \frac{D_{q}^{k} f(0)}{[k]_{q} !} t^{k}+t^{n} g(t)
$$

where $g(t)=\frac{1}{[n-1]_{q} !} \int_{0}^{1}(q u ; q)_{(n-1)}\left[D_{q}^{n} f(t u)-D_{q}^{n} f(0)\right] d_{q} u$. It is easy to see that $g$ is continuous on $\left[0,+\infty\left[, g(0)=0\right.\right.$ and $\int_{0}^{\infty} t^{n} e_{q}^{-\alpha t}|g(t)| d_{q} t<\infty$. Then the previous proposition shows

$$
\int_{0}^{\infty} e_{q}^{-x t} t^{n} g(t) d_{q} t=o\left(\frac{1}{x^{n+1}}\right) .
$$

After a simple calculus, we obtain

$$
\begin{aligned}
\int_{0}^{\infty} e_{q}^{-x t} f(t) d_{q} t & =\sum_{k=0}^{n} \frac{D_{q}^{k} f(0)}{[k]_{q} !} \frac{[k]_{q} !}{q^{\frac{k(k+1)}{2}}} \frac{1}{x^{k+1}}+o\left(\frac{1}{x^{n+1}}\right) \\
& =\sum_{k=0}^{n} \frac{D_{q}^{k} f(0)}{q^{\frac{k(k+1)}{2}}} \frac{1}{x^{k+1}}+o\left(\frac{1}{x^{n+1}}\right) .
\end{aligned}
$$

In the two following results, we study the important case $\varphi(t)=-t^{2}$ and we suppose that $1-q^{2} \in \mathbb{R}_{q^{2},+}$.

Proposition 6. Let $f$ be a function defined on $[0,+\infty[$, continuous at 0 and satisfies

$$
\int_{0}^{\infty} t^{n}|f(t)| e_{q^{2}}^{-\alpha t^{2}} d_{q} t<\infty
$$

for some $\alpha>0$ and $n$ integer, then

$$
\lim _{x \rightarrow+\infty} x^{\frac{n+1}{2}} \int_{0}^{\infty} t^{n} f(t) e_{q^{2}}^{-x t^{2}} d_{q} t=\frac{1}{q+1} \frac{\Gamma_{q^{2}}\left(\frac{n+1}{2}\right)}{K_{q^{2}}\left(\frac{n+1}{2}\right)} f(0), \quad \text { in } \quad \mathbb{R}_{q^{2},+} .
$$

Proof. The change of variables rule gives for $x \in \mathbb{R}_{q^{2},+}$,

$$
\begin{aligned}
\int_{0}^{\infty} e_{q^{2}}^{-x t^{2}} t^{n} d_{q} t & =\frac{1}{1+q} \int_{0}^{\infty} e_{q^{2}}^{-x t} t^{\frac{n-1}{2}} d_{q^{2}} t \\
& =\frac{1}{x^{\frac{n+1}{2}}} \frac{1}{1+q} \frac{\Gamma_{q^{2}}\left(\frac{n+1}{2}\right)}{K_{q^{2}}\left(\frac{n+1}{2}\right)},
\end{aligned}
$$

where $K_{q^{2}}$ is given by (2.24). The rest of the proof can be obtained in the same way of Proposition 5.

Similarly to Corollary 1, the previous proposition and the $q$-Taylor formula give the following result. 
Corollary 2. If $f$ is n-times continuously q-differentiable on $[0,+\infty[$, satisfying the following condition

$$
\int_{0}^{\infty} e_{q^{2}}^{-\alpha t^{2}}|f(t)| d_{q} t<\infty
$$

for some $\alpha>0$, then for all $x \in \mathbb{R}_{q^{2},+}$,

$$
\int_{0}^{\infty} e_{q^{2}}^{-x t^{2}} f(t) d_{q} t=\frac{1}{1+q} \sum_{k=0}^{n} \frac{\Gamma_{q^{2}}\left(\frac{k+1}{2}\right)}{K_{q^{2}}\left(\frac{k+1}{2}\right)} \frac{D_{q}^{k} f(0)}{[k]_{q} !} \frac{1}{x^{\frac{k+1}{2}}}+o\left(\frac{1}{x^{\frac{n+1}{2}}}\right) .
$$

The following proposition is a $q$-analogue of the Watson lemma and it is a simple and useful result to derive asymptotic expansion of Laplace $q$-integrals.

Proposition 7. Let $f$ be a function defined on $[0,+\infty[$ having the asymptotic expansion

$$
f(t) \sim \sum_{n=0}^{\infty} a_{n} t^{\frac{n+\lambda-\mu}{\mu}}, \quad \text { as } \quad t \rightarrow 0,
$$

with $\lambda>0$ and $\mu>0$. Then, provided the q-integral converges for all sufficiently large $x$, we have

$$
\int_{0}^{\infty} f(t) e_{q}^{-x t} d_{q} t \sim \sum_{n=0}^{\infty} \frac{\Gamma_{q}\left(\frac{n+\lambda}{\mu}\right)}{K_{q}\left(\frac{n+\lambda}{\mu}\right)} \frac{a_{n}}{x^{\frac{n+\lambda}{\mu}}}, \quad \text { as } \quad x \rightarrow+\infty \quad \text { in } \quad \mathbb{R}_{q,+} .
$$

Proof. For a positive integer $N$ and a positive real $t$, we put

$$
f_{N}(t)=f(t)-\sum_{n=0}^{N-1} a_{n} t^{\frac{n+\lambda-\mu}{\mu}} .
$$

Since $f_{N}(t)=O\left(t^{\frac{N+\lambda-\mu}{\mu}}\right)$, there exist constants $C_{N}$ and $t_{N}=q^{r} \in \mathbb{R}_{q,+}$ such that

$$
\left|f_{N}(t)\right| \leq C_{N} t^{\frac{N+\lambda-\mu}{\mu}}, \quad \text { for } 0<t<t_{N} .
$$

Then for $x \in \mathbb{R}_{q,+}$,

$$
\begin{aligned}
\left|\int_{0}^{t_{N}} f_{N}(t) e_{q}^{-x t} d_{q} t\right| & \leq C_{N} \int_{0}^{t_{N}} t^{\frac{N+\lambda}{\mu}-1} e_{q}^{-x t} d_{q} t \\
& \leq C_{N} \frac{\Gamma_{q}\left(\frac{N+\lambda}{\mu}\right)}{K_{q}\left(\frac{N+\lambda}{\mu}\right)} \frac{1}{x^{\frac{N+\lambda}{\mu}}} .
\end{aligned}
$$

By hypothesis, $\int_{0}^{\infty}|f(t)| e_{q}^{-x t} d_{q} t$ exists for all sufficiently large $x$, let $X=q^{-p}(1-q)^{-1}$ such an element. So, for all $x=q^{-k}(1-q)^{-1} \in \mathbb{R}_{q,+}$, with $k \geq \max (r, p)$, the $q$-integral $\int_{0}^{\infty}\left|f_{N}(t)\right| e_{q}^{-x t} d_{q} t$ exists and we have

$$
\begin{aligned}
\int_{t_{N}}^{\infty}\left|f_{N}(t)\right| e_{q}^{-x t} d_{q} t & =(1-q) \sum_{n=-\infty}^{r}\left|f_{N}\left(q^{n}\right)\right| e_{q}^{-x q^{n}} q^{n} \\
& =(1-q) \sum_{n=-\infty}^{r}\left|f_{N}\left(q^{n}\right)\right| \frac{q^{n}}{\left(-q^{n-k} ; q\right)_{\infty}} .
\end{aligned}
$$


Since for all integer $n \leq r$, we have

$$
\begin{aligned}
\left(-q^{n-k} ; q\right)_{\infty} & =\left(-q^{n-p} ; q\right)_{\infty}\left(-q^{n-k} ; q\right)_{k-p} \\
& \geq\left(-X(1-q) q^{n} ; q\right)_{\infty}\left(-q^{r-k} ; q\right)_{k-p} \\
& \geq\left(-X(1-q) q^{n} ; q\right)_{\infty} q^{-\frac{(k-r)(k-r+1)}{2}} \\
& \geq\left(-X(1-q) q^{n} ; q\right)_{\infty} q^{-\frac{k^{2}}{2}} q^{-k r} \\
& \geq\left(-X(1-q) q^{n} ; q\right)_{\infty}[x(1-q)]^{r} q^{-\frac{1}{2}\left(\frac{\log (1-q) x}{\log q}\right)^{2}}
\end{aligned}
$$

then

$$
\int_{t_{N}}^{\infty}\left|f_{N}(t)\right| e_{q}^{-x t} d_{q} t \leq[(1-q) x]^{-r} q^{\frac{1}{2}\left(\frac{\log (1-q) x}{\log q}\right)^{2}} \int_{0}^{\infty}\left|f_{N}(t)\right| e_{q}^{-X t} d_{q} t .
$$

Together (4.13) and (4.14) yield

$$
\begin{aligned}
\left|\int_{0}^{\infty} f_{N}(t) e_{q}^{-x t} d_{q} t\right| \leq & C_{N} \frac{\Gamma_{q}\left(\frac{N+\lambda}{\mu}\right)}{K_{q}\left(\frac{N+\lambda}{\mu}\right)} \frac{1}{x^{\frac{N+\lambda}{\mu}}}+ \\
& +[(1-q) x]^{-r} q^{\frac{1}{2}\left(\frac{\log (1-q) x}{\log q}\right)^{2}} \int_{0}^{\infty}\left|f_{N}(t)\right| e_{q}^{-X t} d_{q} t .
\end{aligned}
$$

Which, since $q^{\frac{1}{2}\left(\frac{\log (1-q) x}{\log q}\right)^{2}}=o\left(x^{-v}\right)$ as $x \rightarrow+\infty$ for any positive $v$, establishes the asymptotic expansion for $F(x)$.

By application of the change of variables rule, we obtain

Corollary 3. If $f$ is a function defined on $[0,+\infty[$, having the following asymptotic expansion

$$
f(t) \sim \sum_{n=0}^{\infty} a_{n} t^{n}, \quad \text { as } \quad t \rightarrow 0
$$

then, for $\alpha>0$ and $\beta>0$, we have provided the q-integral converges for all sufficiently large $x$

$$
\int_{0}^{\infty} t^{\alpha-1} f(t) e_{q}^{-x t^{\beta}} d_{q^{\frac{1}{\beta}}} t \sim\left[\frac{1}{\beta}\right] \sum_{q=0}^{\infty} a_{n} \frac{\Gamma_{q}\left(\frac{n+\alpha}{\beta}\right)}{K_{q}\left(\frac{n+\alpha}{\beta}\right)} \frac{1}{x^{\frac{n+\alpha}{\beta}}}, \quad \text { as } \quad x \rightarrow+\infty \quad \text { in } \quad \mathbb{R}_{q,+} .
$$

\section{Fourier $q$-integrals}

The $q$-integral of Fourier type has the form

$$
F(x)=\int_{0}^{\beta} f(t) e_{q}^{i x \varphi(t)} d_{q} t
$$

where $\varphi$ is a real function and we assume $x$ to be a large parameter, and we are interested in the asymptotic behavior of $F(x)$ as $x \rightarrow+\infty$. We start this section by the following useful result, in which we suppose that $1-q \in \mathbb{R}_{q,+}$ : 
Lemma 2. If $\Re(r)>0$, then

$$
\int_{0}^{\infty} t^{r-1} e_{q}^{i t} d_{q} t=\Gamma_{q}(r) \frac{1+i}{\left(-1 ; q^{2}\right)_{\infty}}\left(i q^{r},-i q^{1-r} ; q\right)_{\infty} .
$$

Proof. Using the Ramanujan's sum for ${ }_{1} \psi_{1}(a ; b ; q, z)$ (see [8] and [11]),

$$
\begin{aligned}
\int_{0}^{\infty} t^{r-1} e_{q}^{\frac{i t}{1-q}} d_{q} t & =(1-q) \sum_{n=-\infty}^{\infty} \frac{q^{r n}}{\left(i q^{n} ; q\right)_{\infty}}=\frac{1-q}{(i ; q)_{\infty}} \sum_{n=-\infty}^{\infty}(i ; q)_{n} q^{r n} \\
& =\frac{1-q}{(i ; q)_{\infty}}{ }_{1} \psi_{1}\left(i ; 0 ; q, q^{r}\right)=\frac{1-q}{(i ; q)_{\infty}} \frac{\left(q, i q^{r},-i q^{1-r} ; q\right)_{\infty}}{\left(q^{r},-i q ; q\right)_{\infty}} \\
& =\frac{(1-q)(1+i)}{\left(-1 ; q^{2}\right)_{\infty}} \frac{(q ; q)_{\infty}}{\left(q^{r} ; q\right)_{\infty}}\left(i q^{r},-i q^{1-r} ; q\right)_{\infty} .
\end{aligned}
$$

Since $1-q \in \mathbb{R}_{q,+}$, the change of variable $u=t(1-q)^{-1}$ completes the proof.

In the two following results, we study the case $\varphi(t)=t^{2}$ and we suppose that $1-q \in \mathbb{R}_{q,+}$.

Proposition 8. Let $\beta \in \mathbb{R}_{q^{\frac{1}{2}},+}$, then

$$
\int_{0}^{\beta} e_{q}^{i x t^{2}} d_{q^{\frac{1}{2}}} t=\Gamma_{q}\left(\frac{1}{2}\right) \frac{1+i}{1+q} \frac{\left(-q ; q^{2}\right)_{\infty}}{\left(-1 ; q^{2}\right)_{\infty}} \frac{1}{\sqrt{x}}+O\left(\frac{1}{x}\right) .
$$

Proof. The change of variable $t=u^{\frac{1}{2}}$ leads to

$$
\begin{aligned}
\int_{0}^{\beta} e_{q}^{i x t^{2}} d_{q^{\frac{1}{2}}} t & =\frac{1}{1+q} \int_{0}^{\beta^{2}} e_{q}^{i x u} \frac{d_{q} u}{\sqrt{u}} \\
& =\frac{1}{1+q} \int_{0}^{\infty} e_{q}^{i x u} \frac{d_{q} u}{\sqrt{u}}-\frac{1}{1+q} \int_{\beta^{2}}^{\infty} e_{q}^{i x u} \frac{d_{q} u}{\sqrt{u}} .
\end{aligned}
$$

The proof will be completed by using Lemma 2 and the following result: for a sufficiently large $x \in \mathbb{R}_{q,+}$, we have

$$
\left|\int_{x}^{\infty} e_{q}^{i u} \frac{d_{q} u}{\sqrt{u}}\right| \leq \frac{[2]_{q^{\frac{1}{2}}}}{\sqrt{x}} .
$$

To prove this result let $r \in \mathbb{N}$ such that $1-q=q^{r}$ and let $x=q^{-N}$ with $N>2 r$. We have

$$
\int_{x}^{\infty} e_{q}^{i u} \frac{d_{q} u}{\sqrt{u}}=(1-q) \sum_{n=N}^{\infty} e_{q}^{i q^{-n}} q^{-\frac{n}{2}} .
$$

For $n \geq N$, we have

$$
\begin{aligned}
\left|e_{q}^{i q^{-n}}\right|^{2} & =\frac{1}{\prod_{k=0}^{\infty}\left[1+q^{2(k-n)}(1-q)^{2}\right]}=\frac{1}{\prod_{k=0}^{n-1}\left[1+q^{2(k-n)}(1-q)^{2}\right]} \frac{1}{\left(-(1-q)^{2} ; q^{2}\right)_{\infty}} \\
& =\frac{q^{n^{2}+n}}{(1-q)^{2 n}\left(-q^{2}(1-q)^{-2} ; q^{2}\right)_{n}\left(-(1-q)^{2} ; q^{2}\right)_{\infty}} \\
& \leq \frac{q^{n^{2}+n}}{(1-q)^{2 n}} .
\end{aligned}
$$


Then for all $n \geq N$, we have

$$
\left|e_{q}^{i q^{-n}} q^{-\frac{n}{2}}\right|^{2} \leq \frac{q^{n^{2}}}{(1-q)^{2 n}}=q^{n(n-2 r)} \leq q^{n}
$$

and

$$
\left|e_{q}^{i q^{-n}} q^{-\frac{n}{2}}\right| \leq q^{\frac{n}{2}}
$$

Thus

$$
\left|\int_{x}^{\infty} e_{q}^{i u} \frac{d_{q} u}{\sqrt{u}}\right|=(1-q)\left|\sum_{n=N}^{\infty} e_{q}^{i q^{-n}} q^{-\frac{n}{2}}\right| \leq\left(1+q^{\frac{1}{2}}\right) q^{\frac{N}{2}}=\frac{[2]_{q^{\frac{1}{2}}}}{\sqrt{x}}
$$

Finally, the change of variable $t=x u$ yields

$$
\int_{\beta^{2}}^{\infty} e_{q}^{i x u} \frac{d_{q} u}{\sqrt{u}}=\frac{1}{\sqrt{x}} \int_{x \beta^{2}}^{\infty} e_{q}^{i t} \frac{d_{q} t}{\sqrt{t}} .
$$

Then, for sufficiently large $x \in \mathbb{R}_{q,+}$,

$$
\left|\int_{\beta^{2}}^{\infty} e_{q}^{i x u} \frac{d_{q} u}{\sqrt{u}}\right| \leq \frac{[2]_{q^{\frac{1}{2}}}}{\beta x} .
$$

Proposition 9. Let $\beta \in \mathbb{R}_{q^{\frac{1}{2}},+}$ and $f$ be a function two times continuously differentiable in $[0, \beta]$. Then for $x \in \mathbb{R}_{q,+}$,

$$
\int_{0}^{\beta} f(t) e_{q}^{i x t^{2}} d_{q^{\frac{1}{2}}} t=\Gamma_{q}\left(\frac{1}{2}\right) \frac{1+i}{1+q} \frac{\left(-q ; q^{2}\right)_{\infty}}{\left(-1 ; q^{2}\right)_{\infty}} \frac{f(0)}{\sqrt{x}}+O\left(\frac{1}{x}\right) .
$$

Proof. We can write

$$
f(t)=f(0)+t g(t)
$$

with $g$ is continuously differentiable in $[0, \beta]$. Then

$$
\int_{0}^{\beta} f(t) e_{q}^{i x t^{2}} d_{q^{\frac{1}{2}}} t=f(0) \int_{0}^{\beta} e_{q}^{i x t^{2}} d_{q^{\frac{1}{2}}} t+\int_{0}^{\beta} t g(t) e_{q}^{i x t^{2}} d_{q^{\frac{1}{2}}} t .
$$

Since, $t e_{q}^{i x t^{2}}=\left[\frac{1}{2}\right]_{q} \frac{1}{i x} D_{q^{\frac{1}{2}}}\left(e_{q}^{i x t^{2}}\right)$, a $q$-integration by parts gives

$$
\begin{aligned}
\int_{0}^{\beta} t g(t) e_{q}^{i x t^{2}} d_{q^{\frac{1}{2}}} t & =\left[\frac{1}{2}\right]_{q} \frac{1}{i x}\left[e_{q}^{i x \beta^{2}} g(\beta)-g(0)-\int_{0}^{\beta} D_{q^{\frac{1}{2}}}(g)(t) e_{q}^{i x q t^{2}} d_{q^{\frac{1}{2}}} t\right] \\
& =O\left(\frac{1}{x}\right) .
\end{aligned}
$$

Which completes the proof. 
Let us now assume that $f$ has an asymptotic expansion of the form

$$
f(t) \sim \sum_{k=0}^{\infty} a_{k} t^{\lambda_{k}-1}, \quad \text { as } \quad t \rightarrow 0
$$

where $\Re\left(\lambda_{0}\right)>0$ and $\Re\left(\lambda_{k+1}\right)>\Re\left(\lambda_{k}\right)$ for $k=0,1,2, \ldots$

We suppose that $f$ satisfies the following conditions:

1. $f$ is $m$-times continuously $q$-differentiable in $\left[0,+\infty\left[, m \in \mathbb{N}^{*}\right.\right.$.

2. For all $j \in\{0,1,2, \ldots, m\}, D_{q}^{(j)} f$ is $q$-integrable on $[0,+\infty[$.

We state the following result

Theorem 1. Let $n$ be the smallest nonnegative integer such that $\Re\left(\lambda_{n}\right)>m$. Then

$$
\int_{0}^{\infty} f(t) e_{q}^{i x t} d_{q} t=\sum_{k=0}^{n-1} \frac{b_{k}}{x^{\lambda_{k}}}+o\left(x^{-m}\right), \quad \text { as } \quad x \rightarrow+\infty \quad \text { in } \quad \mathbb{R}_{q,+},
$$

where $b_{k}=\Gamma_{q}\left(\lambda_{k}\right) \frac{1+i}{\left(-1 ; q^{2}\right)_{\infty}}\left(i q^{\lambda_{k}},-i q^{1-\lambda_{k}} ; q\right)_{\infty} a_{k}$.

Proof. Define the function $f_{n}$ by

$$
f(t)=\sum_{k=0}^{n-1} a_{k} t^{\lambda_{k}-1}+f_{n}(t)
$$

Observe that for all $j=0,1,2, \ldots, m$,

$$
D_{q}^{(j)} f_{n}(t)=O\left(t^{\lambda_{n}-j-1}\right), \quad \text { as } \quad t \rightarrow 0,
$$

which implies since $\Re\left(\lambda_{n}\right)>m, D_{q}^{(m)} f_{n}$ is $q$-integrable on $[0,1]$ and

$$
D_{q}^{(j)} f_{n}(0)=0, \quad j=0,1,2, \ldots, m-1 .
$$

The equation (5.5) and Lemma 2 give

$$
\begin{aligned}
\int_{0}^{\infty} f(t) e_{q}^{i x t} d_{q} t & =\sum_{k=0}^{n-1} a_{k} \int_{0}^{\infty} t^{\lambda_{k}-1} e_{q}^{i x t} d_{q} t+\int_{0}^{\infty} f_{n}(t) e_{q}^{i x t} d_{q} t \\
& =\sum_{k=0}^{n-1} \frac{b_{k}}{x^{\lambda_{k}}}+\int_{0}^{\infty} f_{n}(t) e_{q}^{i x t} d_{q} t
\end{aligned}
$$

The condition 2) and the equation (5.5) give

$$
\lim _{t \rightarrow+\infty} D_{q}^{(j)} f_{n}(t) e_{q}^{i x t}=0, \quad j=0,1,2, \ldots, m-1,
$$


for all $x>0$. Then by $q$-integrations by parts we obtain

$$
\begin{aligned}
\int_{0}^{\infty} f_{n}(t) e_{q}^{i x t} d_{q} t & =\frac{i}{x} \int_{0}^{\infty} D_{q} f_{n}(t) e_{q}^{i q x t} d_{q} t \\
& =q^{-\frac{m(m-1)}{2}}\left(\frac{i}{x}\right)^{m} \int_{0}^{\infty} D_{q}^{(m)} f_{n}(t) e_{q}^{i q^{m} x t} d_{q} t
\end{aligned}
$$

Note that

$$
D_{q}^{(m)} f_{n}(t)=D_{q}^{(m)} f(t)-\sum_{k=0}^{n-1} c_{k} t^{\lambda_{k}-m-1},
$$

where $c_{k}=a_{k} \frac{\Gamma_{q}\left(\lambda_{k}\right)}{\Gamma_{q}\left(\lambda_{k}-m\right)}, \quad k=0,1, \ldots, n-1$. Then

$$
\begin{gathered}
\int_{0}^{\infty} D_{q}^{(m)} f_{n}(t) e_{q}^{i q^{m} x t} d_{q} t=\int_{0}^{1} D_{q}^{(m)} f_{n}(t) e_{q}^{i q^{m} x t} d_{q} t+\int_{1}^{\infty} D_{q}^{(m)} f_{n}(t) e_{q}^{i q^{m} x t} d_{q} t \\
=\int_{0}^{1} D_{q}^{(m)} f_{n}(t) e_{q}^{i q^{m} x t} d_{q} t+ \\
+\int_{1}^{\infty}\left(D_{q}^{(m)} f(t)-\sum_{k=0}^{n-2} c_{k} t^{\lambda_{k}-m-1}\right) e_{q}^{i q^{m} x t} d_{q} t-c_{n-1} \int_{1}^{\infty} t^{\lambda_{n-1}-m-1} e_{q}^{i q^{m} x t} d_{q} t
\end{gathered}
$$

The definition of the integer $n$ and Lemma 1 imply that the two first $q$-integrals in the right hand side of the above equality tend to 0 as $x$ tends to $\infty$. Also, since for all $x>0, t>1$, we have

$$
\left|t^{\lambda_{n-1}-m-1} e_{q}^{i q^{m} x t}\right| \leq \frac{1}{(1-q) q^{m} x t^{2}},
$$

then

$$
\lim _{x \rightarrow+\infty} \int_{1}^{\infty} t^{\lambda_{n-1}-m-1} e_{q}^{i q^{m} x t} d_{q} t=0 .
$$

\section{$6 \quad q$-Hankel transform}

The third $q$-Bessel function is defined by (see [9] and [14])

$$
J_{\alpha}\left(x ; q^{2}\right)=x^{\alpha} \frac{\left(q^{2 \alpha+2} ; q^{2}\right)_{\infty}}{\left(q^{2} ; q^{2}\right)_{\infty}}{ }_{1} \varphi_{1}\left(0 ; q^{2 \alpha+2} ; q^{2} ; q^{2} x^{2}\right) .
$$

Taking into account $(2.26),(2.27)$ and (2.28), one can easily prove (see [6] and [14]), that for $x=q^{-n}, n \in \mathbb{N}$,

$$
J_{\alpha}\left(x ; q^{2}\right)=\frac{q^{-n \alpha}}{\left(q^{2} ; q^{2}\right)_{\infty}}\left(q^{2(1-n)} ; q^{2}\right)_{\infty 1} \varphi_{1}\left(0 ; q^{2(1-n)} ; q^{2} ; q^{2 \alpha+2}\right),
$$

which is majorized by

$$
q^{n(n+\alpha+1)} \frac{\left(-q^{2} ; q^{2}\right)_{\infty}\left(-q^{2(\alpha+1)} ; q^{2}\right)_{\infty}}{\left(q^{2} ; q^{2}\right)_{\infty}} .
$$

We summarize some properties of $J_{\alpha}\left(x ; q^{2}\right)$ in the following lemma: 
Lemma 3. (see [6] and [14]) Suppose that $\alpha>-1$, then

1. $J_{\alpha}\left(x ; q^{2}\right) \sim x^{\alpha} \frac{\left(q^{2 \alpha+2} ; q^{2}\right)_{\infty}}{\left(q^{2} ; q^{2}\right)_{\infty}}$ as $x \rightarrow 0$.

2. $\forall x \in \mathbb{R}_{q,+}, \quad\left|J_{\alpha}\left(x ; q^{2}\right)\right| \leq \frac{\left(-q^{2} ; q^{2}\right)_{\infty}\left(-q^{2(\alpha+1)} ; q^{2}\right)_{\infty}}{\left(q^{2} ; q^{2}\right)_{\infty}}\left\{\begin{array}{cll}1 & \text { if } & x \leq 1 \\ q^{\left(\frac{\log x}{\log q}\right)^{2}} & \text { if } & x>1 .\end{array}\right.$

3. $\forall \nu \in \mathbb{R}, J_{\alpha}\left(x ; q^{2}\right)=o\left(x^{-\nu}\right) \quad$ as $\quad x \rightarrow+\infty \quad$ in $\quad \mathbb{R}_{q,+}$.

4. if $f$ is q-integrable on $[0,+\infty[$, we have

$$
\lim _{x \rightarrow+\infty} \int_{0}^{\infty} f(t) J_{\alpha}\left(x t ; q^{2}\right) d_{q} t=0 \quad \text { in } \quad \mathbb{R}_{q,+} .
$$

As in Sec. 5, we shall again assume that $f$ is $m$ times continuously $q$-differentiable on ] $0,+\infty\left[, m \in \mathbb{N}^{*}\right.$, such that for all $0 \leq j \leq m, D_{q}^{j} f$ is $q$-integrable on $[1,+\infty[$ and it has the following asymptotic expansion

$$
f(t) \sim \sum_{k=0}^{\infty} a_{k} t^{\lambda_{k}-1} \quad \text { as } \quad t \rightarrow 0
$$

where $\Re\left(\lambda_{0}+\alpha\right)>0$ and $\Re\left(\lambda_{k+1}\right)>\Re\left(\lambda_{k}\right)$ for $k=0,1,2, \ldots$

We establish the following proposition:

Proposition 10. Let $\alpha \in \mathbb{C}$ such that $\Re(\alpha)>-1$ and suppose that $\Re\left(\lambda_{0}+\alpha\right)>0$. If $n$ is the smallest positive integer such that $\Re\left(\lambda_{n}\right)>m$, then

$$
\int_{0}^{\infty} f(t) J_{\alpha}\left(x t ; q^{2}\right) d_{q} t=\sum_{k=0}^{n-1} \frac{b_{k}}{x^{\lambda_{k}}}+o\left(x^{-m}\right), \quad x \in \mathbb{R}_{q,+},
$$

where $b_{k}=(1+q)\left(1-q^{2}\right)^{\lambda_{k}-1} \frac{\Gamma_{q^{2}}\left(\frac{\lambda_{k}+\alpha}{2}\right)}{\Gamma_{q^{2}}\left(\frac{\alpha+2-\lambda_{k}}{2}\right)} a_{k}$.

Proof. Define the function $f_{n}$ by

$$
f(t)=\sum_{k=0}^{n-1} a_{k} t^{\lambda_{k}-1}+f_{n}(t)
$$

and the functions $f_{n, j}, j=0, \ldots, m$ by

$$
f_{n, 0}(t)=f_{n}(t) \quad \text { and } \quad f_{n, j+1}(t)=[\alpha+j+1]_{q} \frac{f_{n, j}(t)}{t}-D_{q} f_{n, j}(t) .
$$

By induction, we prove that there are constants $\left(C_{j, i}\right)_{0 \leq i \leq j \leq m}$ such that

$$
f_{n, j}(t)=\sum_{i=0}^{j} C_{j, i} \frac{D_{q}^{i} f_{n}(t)}{t^{j-i}}, \quad 0 \leq j \leq m .
$$


Since $f_{n}(t)=f(t)-\sum_{k=0}^{n-1} a_{k} t^{\lambda_{k}-1}=O\left(t^{\lambda_{n}-1}\right)$ as $t \rightarrow 0$, we have for all $j=0,1, \ldots, m$, $f_{n, j}(t)=O\left(t^{\lambda_{n}-j-1}\right)$ as $t \rightarrow 0$. In particular $f_{n, m}$ is $q$-integrable on $[0,1]$ and for all $0 \leq j \leq m-1$ and $x \in \mathbb{R}_{q,+}, \lim _{t \rightarrow 0} f_{n, j}(t) J_{\alpha+j+1}\left(x t ; q^{2}\right)=0$.

We have also,

$$
f_{n, j}(t)=\sum_{i=0}^{j} C_{j, i} \frac{D_{q}^{i} f(t)}{t^{j-i}}-\sum_{k=0}^{n-1} d_{j, k} t^{\lambda_{k}-j-1}, \quad 0 \leq j \leq m,
$$

where $d_{j, k}$ are complex constants. The $q$-integrability of $D_{q}^{j} f$ on $[1,+\infty[$ and the relation 3 ) of Lemma 3 yield for $\nu>0$ and $x \in \mathbb{R}_{q,+}$,

$$
f_{n, j}(t) J_{\nu}\left(x t ; q^{2}\right) \rightarrow 0 \quad \text { as } \quad t \rightarrow+\infty, \quad 0 \leq j \leq m .
$$

Now, from the identity

$$
D_{q}\left[t^{\nu+1} J_{\nu+1}\left(t ; q^{2}\right)\right]=\frac{t^{\nu+1}}{1-q} J_{\nu}\left(t ; q^{2}\right),
$$

it follows by $m q$-integrations by parts

$$
\int_{0}^{\infty} f_{n}(t) J_{\alpha}\left(x t ; q^{2}\right) d_{q} t=\frac{(1-q)^{m} q^{\frac{-m(m-1)}{2}}}{x^{m}} \int_{0}^{\infty} f_{n, m}(t) J_{\alpha+m}\left(x q^{m} t ; q^{2}\right) d_{q} t .
$$

It remains to prove that the $q$-integral $\int_{0}^{\infty} f_{n, m}(t) J_{\alpha+m}\left(x q^{m} t ; q^{2}\right) d_{q} t$ tends to 0 as $x$ tends to $\infty$ in $\mathbb{R}_{q,+}$.

One can write

$$
\begin{aligned}
\int_{0}^{\infty} f_{n, m}(t) J_{\alpha+m}\left(x q^{m} t ; q^{2}\right) d_{q} t & =\int_{0}^{1} f_{n, m}(t) J_{\alpha+m}\left(x q^{m} t ; q^{2}\right) d_{q} t+ \\
& +\sum_{i=0}^{m} C_{m, i} \int_{1}^{\infty} \frac{D_{q}^{i} f(t)}{t^{m-i}} J_{\alpha+m}\left(x q^{m} t ; q^{2}\right) d_{q} t- \\
& -\sum_{k=0}^{n-1} d_{m, k} \int_{1}^{\infty} t^{\lambda_{k}-m-1} J_{\alpha+m}\left(x q^{m} t ; q^{2}\right) d_{q} t
\end{aligned}
$$

Using the Lebesgue theorem, the relation 4) of Lemma 3, the $q$-integrability of $D_{q}^{j} f$, $0 \leq j \leq m$ on $\left[1,+\infty\left[\right.\right.$ and the $q$-integrability of $f_{n, m}$ on $[0,1]$, we obtain

$$
\int_{0}^{1} f_{n, m}(t) J_{\alpha+m}\left(x q^{m} t ; q^{2}\right) d_{q} t \rightarrow 0 \quad \text { as } \quad x \rightarrow+\infty
$$

and

$$
\int_{1}^{\infty} \frac{D_{q}^{i} f(t)}{t^{m-i}} J_{\alpha+m}\left(x q^{m} t ; q^{2}\right) d_{q} t \rightarrow 0 \quad \text { as } \quad x \rightarrow+\infty, \quad 0 \leq i \leq m .
$$

Now, by using the definition of the integer $n$, we have for all $k \in\{0, \ldots, n-1\}$ and $x, t \in\left[1,+\infty\left[\cap \mathbb{R}_{q,+}\right.\right.$,

$$
\left|t^{\lambda_{k}-m-1} J_{\alpha+m}\left(x q^{m} t ; q^{2}\right)\right| \leq t^{-1}\left|J_{\alpha+m}\left(x q^{m} t ; q^{2}\right)\right| \leq \frac{C_{\alpha, m}}{x} \frac{1}{t^{2}} .
$$


where $C_{\alpha, m}=\frac{\left(-q^{2} ; q^{2}\right)_{\infty}\left(-q^{2(\alpha+m+1)} ; q^{2}\right)_{\infty}}{\left(q^{2} ; q^{2}\right)_{\infty}}$.

Which proves that for all $k \in\{0, \ldots, n-1\}, \int_{1}^{\infty} t^{\lambda_{k}-m-1} J_{\alpha+m}\left(x q^{m} t ; q^{2}\right) d_{q} t \rightarrow 0$ as $x \rightarrow+\infty$.

Thus

$$
\int_{0}^{\infty} f_{n, m}(t) J_{\alpha+m}\left(x q^{m} t ; q^{2}\right) d_{q} t \rightarrow 0 \quad \text { as } \quad x \rightarrow+\infty \quad\left(\mathrm{in} \mathbb{R}_{q,+}\right)
$$

and $\int_{0}^{\infty} f_{n}(t) J_{\alpha}\left(x t ; q^{2}\right) d_{q} t=o\left(x^{-m}\right)$.

By integration of the equality (6.4) on $[0,+\infty[$ and the fact (see [5])

$$
\int_{0}^{\infty} t^{\lambda-1} J_{\alpha}\left(t ; q^{2}\right) d_{q}=(1+q)\left(1-q^{2}\right)^{\lambda-1} \frac{\Gamma_{q^{2}}\left(\frac{\lambda+\alpha}{2}\right)}{\Gamma_{q^{2}}\left(\frac{\alpha+2-\lambda}{2}\right)}, \Re(\lambda)>-\Re(\alpha),
$$

we have

$$
\int_{0}^{\infty} f(t) J_{\alpha}\left(x t ; q^{2}\right) d_{q}=\sum_{k=0}^{n-1} \frac{b_{k}}{x^{\lambda_{k}}}+o\left(x^{-m}\right) .
$$

Additionally, if $\frac{\log (1-q)}{\log (q)} \in \mathbb{Z}$, then from the well-known relations (see [5], [14])

$$
\cos \left(x ; q^{2}\right)=\frac{\Gamma_{q^{2}}\left(\frac{1}{2}\right)}{q\left(1+q^{-1}\right)^{\frac{1}{2}}} x^{\frac{1}{2}} J_{-\frac{1}{2}}\left(\frac{1-q}{q} x ; q^{2}\right)
$$

and

$$
\sin \left(x ; q^{2}\right)=\frac{\Gamma_{q^{2}}\left(\frac{1}{2}\right)}{\left(1+q^{-1}\right)^{\frac{1}{2}}} x^{\frac{1}{2}} J_{\frac{1}{2}}\left(\frac{1-q}{q} x ; q^{2}\right),
$$

one can deduce easily the asymptotic behavior of the $q$-Cosine and the $q$-Sine Fourier transforms.

Proposition 11. Let $n$ be the smallest positive integer such that $\Re\left(\lambda_{n}\right)+\frac{1}{2}>m$, then for $x \in \mathbb{R}_{q,+}$, we have

1. If $\Re\left(\lambda_{0}\right)>0$, then

$$
\begin{aligned}
\int_{0}^{\infty} f(t) \cos \left(x t ; q^{2}\right) d_{q} t & =\sum_{k=0}^{n-1} \frac{\Gamma_{q^{2}}\left(\frac{1}{2}\right)}{\left(1+q^{-1}\right)^{\frac{1}{2}}} q^{\lambda_{k}-\frac{1}{2}}(1+q)^{\lambda_{k}-\frac{1}{2}} \frac{\Gamma_{q^{2}}\left(\frac{\lambda_{k}}{2}\right)}{\Gamma_{q^{2}}\left(\frac{1-\lambda_{k}}{2}\right)} \frac{a_{k}}{x^{\lambda_{k}}}+ \\
& +o\left(x^{-m+\frac{1}{2}}\right) .
\end{aligned}
$$

2. If $\Re\left(\lambda_{0}\right)>-1$, then

$$
\begin{aligned}
\int_{0}^{\infty} f(t) \sin \left(x t ; q^{2}\right) d_{q} t= & \sum_{k=0}^{n-1} \frac{\Gamma_{q^{2}}\left(\frac{1}{2}\right)}{\left(1+q^{-1}\right)^{\frac{1}{2}}} q^{\lambda_{k}+\frac{1}{2}}(1+q)^{\lambda_{k}-\frac{1}{2}} \frac{\Gamma_{q^{2}}\left(\frac{\lambda_{k}+1}{2}\right)}{\Gamma_{q^{2}}\left(\frac{2-\lambda_{k}}{2}\right)} \frac{a_{k}}{x^{\lambda_{k}}}+ \\
& +o\left(x^{-m+\frac{1}{2}}\right) .
\end{aligned}
$$


Example 4. $f(t)=e_{q}^{-t}=\left[((1-q) t ; q)_{\infty}\right]^{-1}$, then $f$ is a $C^{\infty}$ function on $[0,+\infty[$ and for all $n \in \mathbb{N}, D_{q}^{n} f=(-1)^{n} f$. Furthermore, $f$ is $q$-integrable on $[0,+\infty[$ and we have

$$
f(t) \sim \sum_{n=0}^{\infty} \frac{(-1)^{n}}{[n]_{q} !} t^{n} \quad \text { as } \quad t \rightarrow 0 .
$$

So, for all $m \in \mathbb{N}$, we have

- when $\alpha>-1$,

$$
\begin{aligned}
\int_{0}^{\infty} f(t) J_{\alpha}\left(x t ; q^{2}\right) d_{q} t= & \sum_{n=0}^{m} \frac{(-1)^{n}(1+q)\left(1-q^{2}\right)^{n}}{[n]_{q} !} \frac{\Gamma_{q^{2}}\left(\frac{1+n+\alpha}{2}\right)}{\Gamma_{q^{2}}\left(\frac{1-n+\alpha}{2}\right)} \frac{1}{x^{n}}+ \\
& +o\left(x^{-m-1}\right) .
\end{aligned}
$$

- If we add $1-q \in \mathbb{R}_{q,+}$,

$$
\begin{aligned}
\int_{0}^{\infty} f(t) \cos \left(x t ; q^{2}\right) d_{q} t & =\sum_{n=0}^{m} \frac{\Gamma_{q^{2}}\left(\frac{1}{2}\right)}{\left(1+q^{-1}\right)^{\frac{1}{2}}} \frac{(-1)^{n} q^{n+\frac{1}{2}}(1+q)^{n+\frac{1}{2}}}{[n]_{q} !} \frac{\Gamma_{q^{2}}\left(\frac{n+1}{2}\right)}{\Gamma_{q^{2}}\left(\frac{-n}{2}\right)} \frac{1}{x^{n+1}}+ \\
& +o\left(x^{-m-\frac{3}{2}}\right)
\end{aligned}
$$

and

$$
\begin{aligned}
\int_{0}^{\infty} f(t) \sin \left(x t ; q^{2}\right) d_{q} t & =\sum_{n=0}^{m} \frac{\Gamma_{q^{2}}\left(\frac{1}{2}\right)}{\left(1+q^{-1}\right)^{\frac{1}{2}}} \frac{(-1)^{n} q^{n+\frac{3}{2}}(1+q)^{n+\frac{1}{2}}}{[n]_{q} !} \frac{\Gamma_{q^{2}}\left(\frac{n+2}{2}\right)}{\Gamma_{q^{2}}\left(\frac{1-n}{2}\right)} \frac{1}{x^{n+1}}+ \\
& +o\left(x^{-m-\frac{3}{2}}\right) .
\end{aligned}
$$

Example 5. $f(t)=\left(1+t^{2}\right)^{-1}$.

$f$ is a $C^{\infty}$ function on $[0,+\infty$ [ and has the following asymptotic expansion near 0

$$
f(t) \sim \sum_{n=0}^{\infty}(-1)^{n} t^{2 n} \quad \text { as } \quad t \rightarrow 0 .
$$

Besides, by induction, we prove that for $n \in \mathbb{N}, D_{q}^{n} f$ is $q$-integrable on $[1,+\infty[$. Then for all $m \in \mathbb{N}$, we have

$$
\begin{aligned}
\int_{0}^{\infty} \frac{\cos \left(x t ; q^{2}\right)}{1+t^{2}} d_{q} t & =\sum_{n=0}^{m} \frac{\Gamma_{q^{2}}\left(\frac{1}{2}\right)}{\left(1+q^{-1}\right)^{\frac{1}{2}}}(-1)^{n} q^{2 n+\frac{1}{2}}(1+q)^{2 n+\frac{1}{2}} \frac{\Gamma_{q^{2}}\left(\frac{2 n+1}{2}\right)}{\Gamma_{q^{2}}(-n)} \frac{1}{x^{2 n+1}} \\
& +o\left(x^{-2 m-\frac{3}{2}}\right) \\
& =o\left(x^{-2 m-\frac{3}{2}}\right) .
\end{aligned}
$$

Acknowledgments. The authors thank the referee for many helpful suggestions and constructive criticism. He pointed us a reference of the book about quantum calculus and we would like to note that our approach in this paper is very similar to the classical picture developed in [17]. 


\section{References}

[1] De Sole A and Kac V G, On integral representations of $q$-gamma and $q$-beta functions, preprint arXiv:math.QA/0302032.

[2] Ernst T, A new notation for $q$-calculus and a new $q$-Taylor formula, U. U. D. M. Report 1999:25, ISSN 1101-3591, Uppsala University, 1999.

[3] Ernst T, The history of $q$-Calculus and a New Method, U. U. D. M. Report 2000:16, ISSN 1101-3591 Uppsala University, 2000.

[4] Ernst T, A method for q-Calculus, J. Nonlinear Math. Phys. 10 (2003), 487-525.

[5] Fitouhi A, Bettaibi N and Brahim K, The Mellin transform in Quantum Calculus, to appear in Constructive Approximation.

[6] Fitouhi A and Bouzeffour F, $q$-cosine Fourier transform and $q$-heat equation, to appear in Ramanujan Journal.

[7] Fitouhi A, Hamza M M and Bouzeffour F, The $q$ - $J_{\alpha}$ Bessel function, J. Approx. Theory 115 (2002), 144-166.

[8] Gasper G and Rahmen M, Basic hypergeometric series, Encyclopedia of Mathematics and its application 35, Cambridge University Press, Cambridge, 1990.

[9] Ismail $\mathrm{M} \mathrm{E} \mathrm{H,} \mathrm{The} \mathrm{zeros} \mathrm{of} \mathrm{basic} \mathrm{Bessel} \mathrm{functions,} \mathrm{the} \mathrm{function} J_{v+a x}(x)$, and associated orthogonal polynomials, J. Math. Anal. Appl. 86 (1982), 1-19.

[10] Jackson F H, On a q-definite integrals, Q. J. Pure Appl. Math. 41 (1910), 193-203.

[11] Kac V G and Cheung P, Quantum Calculus, Springer-Verlag, New York, 2002.

[12] Koornwinder T H, q-Special Functions, a Tutorial, in Deformation theory and quantum groups with applications to mathematical physics, Contemp. Math. 134, Editors: Gerstenhaber M and Stasheff J, American Mathematical Society, Providence, 1992, 141-142.

[13] Koornwinder T H, Special Functions and q-Commuting Variables, in Special Functions, $q$-Series and related Topics, Fields Inst. Commun. 14, Editors: Ismail M E H, Masson D R and Rahman M, American Mathematical Society, Providence, 1997, 131-166; arXiv:q-alg/9608008.

[14] Koornwinder T H and Swarttouw R F, On $q$-analogues of the Fourier and Hankel transforms, Trans. Amer. Math. Soc. 333 (1992), 445-461.

[15] Moak D S, The q-analogue of Stirlings formula, Rocky Mt. J. Math. 14 (1984), 403-413.

[16] Olde Daalhuis A B, Asymptotic expansions for $q$-Gamma, $q$-exponentials, and $q$-Bessel functions, J. Math. Anal. Appl. 186 (1994), 896-913.

[17] Wong R, Asymptotic approximations of integrals (Computer Science and Scientific Computing), Academic Press, New York, 1989. 